This is an electronic reprint of the original article. This reprint may differ from the original in pagination and typographic detail.

Author(s): Gao, Yan; Haïssinsky, Peter; Meyer, Daniel; Zeng, Jinsong

Title: Invariant Jordan curves of Sierpinski carpet rational maps

Year: $\quad 2018$

Version:

Please cite the original version:

Gao, Y., Haïssinsky, P., Meyer, D., \& Zeng, J. (2018). Invariant Jordan curves of Sierpinski carpet rational maps. Ergodic Theory and Dynamical Systems, 38(2), 583600. https://doi.org/10.1017/etds.2016.47

All material supplied via JYX is protected by copyright and other intellectual property rights, and duplication or sale of all or part of any of the repository collections is not permitted, except that material may be duplicated by you for your research use or educational purposes in electronic or print form. You must obtain permission for any other use. Electronic or print copies may not be offered, whether for sale or otherwise to anyone who is not an authorised user. 


\title{
INVARIANT JORDAN CURVES OF SIERPIŃSKI CARPET RATIONAL MAPS
}

\author{
YAN GAO, PETER HAÏSSINSKY, DANIEL MEYER, AND JINSONG ZENG
}

\begin{abstract}
In this paper, we prove that if $R: \widehat{\mathbb{C}} \rightarrow \widehat{\mathbb{C}}$ is a postcritically finite rational map with Julia set homeomorphic to the Sierpinski carpet, then there is an integer $n_{0}$, such that, for any $n \geq n_{0}$, there exists an $R^{n}$-invariant Jordan curve $\Gamma$ containing the postcritical set of $R$.
\end{abstract}

KEYWORDs: Invariant Jordan curve, rational maps, Sierpiński carpet Julia sets, expanding Thurston maps.

\section{Contents}

1. Introduction

1.1. Outline

1.2. Notation

2. Branched covers, Thurston and rational maps

2.1. Branched coverings

2.2. Thurston maps

2.3. Fatou dynamics

2.4. Orbifold metric

2.5. Sierpiński carpet Julia sets

3. Review of invariant Jordan curves

4. Invariant Jordan curves that subdivide sectors

5. Expanding quotients

6. Lifts of Jordan curves

7. Acknowledgments

References

\section{INTRODUCTION}

A central problem in Complex Dynamics is the classification of rational maps of a given degree up to Möbius conjugation. A general approach to this problem is to structure this space of maps around the so-called postcritically finite rational maps. In the case of postcritically finite polynomials, Douady and Hubbard have introduced the so-called Hubbard trees which capture their dynamical features $[\mathrm{DH}]$. A long-standing problem is to develop analogous combinatorial invariants for general rational maps, cf. [McM1, Problem 5.5]. The first works on this problem concerned degree two rational maps [Wit, Ber, Ree1. For postcritically finite Newton methods, there has also been recent progress [LMS1, LMS2]. In general degree, combinatorial tools have been introduced leading to the construction of rational maps from subdivision rules [CFKP]; see also [CGNPP where the 
authors provide a conjectural picture of critically fixed rational maps from finite graphs, and [Ros] where the author looks for planar graphs characterizing postcritically finite rational maps (the present work is used in the latter).

In $[\mathrm{BM}, \mathrm{CFP}$, the authors prove independently

Theorem 1.1. Let $R: \widehat{\mathbb{C}} \rightarrow \widehat{\mathbb{C}}$ be a postcritically finite rational map with no critical periodic cycle. Then there is an integer $n_{0}$, such that, for any $n \geq n_{0}$, there exists an $R^{n}$-invariant Jordan curve $\Gamma$ containing the postcritical set of $R$.

Here by $\Gamma$ is $R^{n}$-invariant we mean that it is $R^{n}$-forward invariant, i.e., $R^{n}(\Gamma) \subset \Gamma$. The curve $\Gamma$ enables us to introduce a Markov partition and to develop a combinatorial description of the dynamics. Actually, this theorem is proved in the broader setting of expanding Thurston maps in [BM], cf. Theorem 3.1. Building on this work, we shall prove in the present paper

Theorem 1.2. Let $R: \widehat{\mathbb{C}} \rightarrow \widehat{\mathbb{C}}$ be a postcritically finite rational map with Julia set homeomorphic to the Sierpinski carpet. Then there is an integer $n_{0}$, such that, for any $n \geq n_{0}$, there exists an $R^{n}$-invariant Jordan curve $\Gamma$ containing the postcritical set of $R$.

The proof actually shows that we may choose $\Gamma$ in any homotopy class relative to the postcritical points. More precisely for any Jordan curve $\widetilde{\Gamma} \subset \widehat{\mathbb{C}}$ with post $(f) \subset \widetilde{\Gamma}$ there is $n_{0}=n_{0}(\widetilde{\Gamma})$ such that for all $n \geq n_{0}$ there is an $R^{n}$-invariant Jordan curve $\Gamma \subset \widehat{\mathbb{C}}$ that is isotopic to $\widetilde{\Gamma}$ relative to the postcritical points (thus contains all postcritical points).

REMARK. In Ree2, Rees constructs a Markov partition for geometrically finite rational maps with Julia sets homeomorphic to the Sierpiński carpet. Her approach is very different.

There are many examples of postcritically finite rational maps with Julia sets homeomorphic to the Sierpiński carpet. The first example is due to Milnor and Tan Lei [Mil1, Appendix]; see also the survey [Dev] and the references therein for many others. These rational maps and their Julia sets play a particular role. These maps are centers of hyperbolic components. Conjecturally, those components are relatively compact in the space of rational functions up to Möbius conjugation [McM1, Question 5.3] (established in degree 2 in Eps]. Moreover, their Julia sets turn out to be rigid in a very strong sense [BLM].

1.1. Outline. The proof of Theorem 1.2 proceeds as follows. Let $R: \widehat{\mathbb{C}} \rightarrow \widehat{\mathbb{C}}$ be a postcritically finite rational map with Sierpiński carpet Julia set. Collapse the closure of each Fatou component. This yields an expanding Thurston map $f: S^{2} \rightarrow S^{2}$ in the sense of [BM] by Theorem 5.1. Using Theorem 3.1, or rather the appropriate version Theorem 4.1. we obtain a Jordan curve $\mathcal{C}$ that is invariant for any sufficiently high iterate $f^{n}$ and whose lift to $\widehat{\mathbb{C}}$ contains an $R^{n}$-invariant Jordan curve, see Theorem 6.1 and its proof.

1.2. Notation. The 2 -sphere is denoted by $S^{2}$, the Riemann sphere by $\widehat{\mathbb{C}}$ and the unit disk by $\mathbb{D}$. The set of critical points of a branched covering map $f$ is denoted by $\operatorname{crit}(f)$, the set of postcritical points by post $(f)$ (see Section 2.1). The Julia set of a rational map $R$ will be denoted by $\mathcal{J}_{R}$; the Fatou set is $\mathcal{F}_{R}$. Given two positive quantities $a$ and $b$, we will write $a \lesssim b$ or $b \gtrsim a$ if there is a constant $C>0$ such that $a \leq C b$. The $\delta$-neighborhood of a set $A$ is denoted by $\mathcal{N}^{\delta}(A)$.

\section{Branched COVERs, Thurston And RATiOnAl MAPS}

Here we present some elementary background that will be used in this paper. More details can be found in [BM] and [HP09]. 
2.1. Branched coverings. A map $f: S^{2} \rightarrow S^{2}$ is a branched covering (of the sphere $\left.S^{2}\right)$ if there are orientation-preserving homeomorphisms $\varphi, \psi: S^{2} \rightarrow \widehat{\mathbb{C}}$ and a rational map $R: \widehat{\mathbb{C}} \rightarrow \widehat{\mathbb{C}}$ such that $f=\psi^{-1} \circ R \circ \varphi$. It is characterized by being finite-to-one, open, and orientation-preserving cf. [Why2, Theorem X.5.1]. The degree $\operatorname{deg} f$ and local degree at $x \operatorname{deg}(f, x)$ of $f$ are defined as the degree of $R$ and the local degree of $R$ at $\varphi(x)$ : $\operatorname{deg}(f, x)=d$ means there are charts in the neighborhoods of $x$ and $f(x)$ so that $f$ takes the form $z \mapsto z^{d}$.

The $n$-th iterate of $f$ will be denoted by $f^{n}$. The set of critical points crit $(f)$ corresponds to the points $c \in S^{2}$ such that $\operatorname{deg}(f, c)>1$, i.e., $R^{\prime}(\varphi(c))=0$ (in a suitable chart). The postcritical set post $(f)$ is defined as

$$
\operatorname{post}(f)=\left\{f^{n}(c): c \in \operatorname{crit}(f), n \geq 1\right\} .
$$

Note that $f(\operatorname{post}(f)) \subset \operatorname{post}(f)$ and $\operatorname{post}(f)=\operatorname{post}\left(f^{n}\right)$ for any $n \geq 1$. A finite branched covering $f$ is postcritically finite if $\operatorname{post}(f)$ is a finite set.

If $f$ is postcritically finite, then every postcritical point $p \in \operatorname{post}(f)$ is preperiodic, i.e., there are minimal iterates $k \geq 0$ and $m \geq 1$ such that $f^{k+m}(p)=f^{k}(p) ; m$ is the period of the cycle $\left\{f^{k}(x), \ldots, f^{k+m-1}(x)\right\}$. If the cycle does not contain a critical point, we say that $p$ is of Julia-type, and we denote by post $\mathcal{J}(f)$ the set of Julia-type postcritical points. Otherwise, the periodic cycle contains a critical point and we say $p$ is of Fatou-type; let $\operatorname{post}_{\mathcal{F}}(f)$ denote the set of postcritical points of Fatou-type. Note that $p \in \operatorname{post}_{\mathcal{F}}(f)$ if and only if $\operatorname{deg}\left(f^{n}, p\right) \rightarrow \infty$ as $n \rightarrow \infty$. The terminology is of course explained by the well-known fact that if $f$ is a rational map then a postcritical point $p$ is of Julia-type if and only if $p$ is contained in the Julia set and is of Fatou-type if and only if $p$ is contained in the Fatou set of $f$.

We mention the following fact for future reference, which is a consequence of the definitions and of the Riemann-Hurwitz formula.

Lemma 2.1. Let $f: S^{2} \rightarrow S^{2}$ be a finite branched covering and $V \subset S^{2}$ be a Jordan domain that contains at most a single critical value $p$. Then every component $U$ of $f^{-1}(V)$ is a Jordan domain and $f: U \rightarrow V$ is equivalent to $z^{d}$, where $d=\operatorname{deg}(f, c)$ and $c$ is the unique preimage of $p$ in $U$, i.e., there are orientation-preserving homeomorphisms $\varphi: U \rightarrow \mathbb{D}$ and $\psi: V \rightarrow \mathbb{D}$ such that $\varphi(c)=\psi(f(c))=0$ and $\psi \circ f \circ \varphi^{-1}: \mathbb{D} \rightarrow \mathbb{D}$ is $z \mapsto z^{d}$.

2.2. Thurston maps. The branched covers of the sphere that we will be considering will not always be rational. In this situation, the following notion is very useful.

Definition 2.2. A Thurston map is an orientation-preserving, postcritically finite, branched covering of the sphere,

$$
f: S^{2} \rightarrow S^{2}
$$

We fix a base metric $\sigma$ on $S^{2}$ that induces the standard topology on $S^{2}$. Consider a Jordan curve $\mathcal{C} \supset \operatorname{post} f$. The Thurston map $f$ is called expanding if

$$
\operatorname{mesh} f^{-n}(\mathcal{C}) \rightarrow 0 \text { as } n \rightarrow \infty \text {. }
$$

where mesh $f^{-n}(\mathcal{C})$ denotes the maximal diameter of a component of $S^{2} \backslash f^{-n}(\mathcal{C})$.

In [BM, Lemma 6.1] it was shown that this definition is independent of the chosen curve $\mathcal{C}$. This notion of "expansion" agrees with the one by Haïssinsky-Pilgrim in [HP09] (see [BM, Proposition 6.3]).

Let $f: S^{2} \rightarrow S^{2}$ be a Thurston map and fix a Jordan curve $\mathcal{C} \subset S^{2}$ with post $(f) \subset \mathcal{C}$. The closure of one of the two components of $S^{2} \backslash \mathcal{C}$ is called a 0-tile. Similarly, we call the 
closure of one component of $S^{2} \backslash f^{-n}(\mathcal{C})$ an $n$-tile (for any $n \in \mathbb{N}_{0}$ ). The set of all $n$-tiles is denoted by $\mathbf{X}^{n}(\mathcal{C})$. For any $n$-tile $X$, the set $f^{n}(X)=X^{0}$ is a 0 -tile and

$$
f^{n}: X \rightarrow X^{0} \text { is a homeomorphism, }
$$

see [BM, Proposition 5.17]. This means in particular that each $n$-tile is a closed Jordan domain. The definition of "expansion" implies that $n$-tiles become arbitrarily small, this is the (only) reason we require expansion.

We call the points in $f^{-n}(\operatorname{post}(f))$ the $n$-vertices, so the postcritical points are exactly the 0 -vertices. The closure of any component of $f^{-n}(\mathcal{C}) \backslash f^{-n}(\operatorname{post}(f))$ is an $n$-edge. Thus the 0-edges are precisely the closed arcs into which the points post $(f) \subset \mathcal{C}$ divide $\mathcal{C}$.

The $n$-tiles, $n$-edges, $n$-vertices form a cell complex when viewed as $2-, 1-$, and 0 -cells (see [BM, Chapter 5]).

If we consider just the $n$-edges and $n$-vertices, we obtain a 1 -dimensional cell complex, i.e., a graph in the natural way. Note that this graph may have multiple edges, but no loop edges.

An $n$-edge path is a path in this graph, meaning that it is a finite sequence $e_{1}, \ldots, e_{N}$ of $n$-edges, where $e_{j} \cap e_{j+1}$ is an $n$-vertex for all $j=1, \ldots, N-1$. Such an $n$-edge path is simple if furthermore $e_{i} \cap e_{j}=\emptyset$ for $|i-j| \geq 2$. We allow an $n$-path to consist of only a single $n$-vertex, which is then simple.

2.3. Fatou dynamics. Let us now assume again that $R: \widehat{\mathbb{C}} \rightarrow \widehat{\mathbb{C}}$ is a postcritically finite rational map. Then each Fatou component $\Omega$ of $R$ is simply connected, cf. [McM2, Remark, p. 35]). From Boettcher's theorem it then follows that there is a conformal map $\eta_{\Omega}: \mathbb{D} \rightarrow \Omega$ and some power $d_{\Omega}$ such that $R \circ \eta_{\Omega}(z)=\eta_{R(\Omega)}\left(z^{d_{\Omega}}\right)$ for all $z \in \mathbb{D}$.

Furthermore, the Julia set $\mathcal{J}_{R}$ of $R$ is locally connected, see [Mil2, Theorem 19.7]. Thus it follows from Carathéodory's theorem that the conformal map $\eta$ extends to a continuous and surjective map $\eta_{\Omega}: \overline{\mathbb{D}} \rightarrow \bar{\Omega}$.

An internal ray is the image $\eta_{\Omega}([0,1) \sigma)$ for some Fatou component $\Omega$ and some complex number of modulus one $\sigma \in \partial \mathbb{D}$. Note that internal rays are mapped to internal rays under $R$.

2.4. Orbifold metric. Again let $R: \widehat{\mathbb{C}} \rightarrow \widehat{\mathbb{C}}$ be a postcritically finite rational map (or rational Thurston map). For any such map $R$, there exists a complete metric $d_{\mathcal{O}}$ called the orbifold metric on $\mathcal{O}=\widehat{\mathbb{C}} \backslash$ post $_{\mathcal{F}}(R)$, the complement of the postcritical points of Fatoutype, with the following properties (see [Mil2, Appendix E] as well as [BM, Appendix A.9]). It is induced by a conformal metric $\rho(z)|d z|$ with $\rho$ smooth in the complement of post $(R)$, and, for any $w \in \mathcal{O}$ and $z \in R^{-1}(w)$, it satisfies $\left\|R^{\prime}(z)\right\|_{\mathcal{O}}>1$. Thus for any compact set $K \subset \mathcal{O}$ there is a constant $\lambda>1$ such that $\left\|R^{\prime}(z)\right\|_{\mathcal{O}} \geq \lambda$ for all $z \in R^{-1}(K)$. See Mil2, Theorem 19.6] for details.

From the previous section it is easy to see that we may choose such a relatively compact set $W \subset \mathcal{O}$ such that $W^{\prime}=R^{-1}(W)$ has compact closure in $W$ and such that $W$ contains all Fatou components that do not contain a Fatou-type postcritical point. Let us then fix the constant $\lambda>1$ for this set $W$ as above.

Lemma 2.3 (Shrinking Lemma). Let $R: \widehat{\mathbb{C}} \rightarrow \widehat{\mathbb{C}}$ be a postcritically finite rational map. There are constants $\delta>0$ and $C>0$ such that the following holds. Let $K \subset \mathcal{O}$ be $a$ compact set intersecting $\mathcal{J}_{R}$ and diameter at most $\delta$, then for any iterate $n \geq 1$ and any component $L$ of $R^{-n}(K)$, the inequality

$$
\operatorname{diam}_{\mathcal{O}}(L) \leq C \operatorname{diam}_{\mathcal{O}}(K) / \lambda^{n}
$$


holds.

Proof. Since $d_{\mathcal{O}}$ comes from a conformal metric, we may find $\chi \geq 1$ and $r_{0}>0$ such that, for any $z \in W$, any $r \in\left(0, r_{0}\right]$, we can find a Jordan domain $D$ such that $B_{\mathcal{O}}(z, r) \subset$ $D \subset B_{\mathcal{O}}(z, \chi r)$. Let $\delta \in\left(0, r_{0}\right]$ be small enough so that, for any distinct postcritical points $z, w$ of Julia type, $d_{\mathcal{O}}(z, w)>\chi^{2} \delta$ and $d_{\mathcal{O}}\left(\mathcal{J}_{R}, \mathcal{O} \backslash W\right)>\chi^{2} \delta$. If $K$ is a compact subset with connected complement of diameter at most $\delta$, then either (a) we can find $z \in K$ and a simply connected domain $D$ such that $B_{\mathcal{O}}\left(z, \operatorname{diam}_{\mathcal{O}} K\right) \subset D \subset B_{\mathcal{O}}\left(z, \chi \operatorname{diam}_{\mathcal{O}} K\right)$ and $B_{\mathcal{O}}\left(z, \chi \operatorname{diam}_{\mathcal{O}} K\right)$ is disjoint from $\operatorname{post}(R)$, or (b) we can find $z \in \operatorname{post}_{\mathcal{J}}(R)$ and a simply connected set $D$ such that $K \subset B_{\mathcal{O}}\left(z, \chi \operatorname{diam}_{\mathcal{O}} K\right) \subset D \subset B_{\mathcal{O}}\left(z, \chi^{2} \operatorname{diam}_{\mathcal{O}} K\right)$ and $B_{\mathcal{O}}\left(z, \chi \operatorname{diam}_{\mathcal{O}} K\right) \cap \operatorname{post}(R)=\{z\}$. Fix $n \geq 1$; according to Lemma 2.1, for any component $D^{\prime}$ of $R^{-n}(D), R^{-n}(\{z\}) \cap D^{\prime}$ is a singleton $\left\{z^{\prime}\right\}$ and, for any $x \in D^{\prime}, d_{\mathcal{O}}\left(x, z^{\prime}\right) \leq$ $d_{\mathcal{O}}\left(R^{n}(x), z\right) / \lambda^{n}$. Therefore, if $L$ is a component of $R^{-n}(K)$, then $\operatorname{diam}_{\mathcal{O}} L \leq 2 \operatorname{diam}_{\mathcal{O}} K / \lambda^{n}$ holds in case (a), and, in case (b), we obtain $\operatorname{diam}_{\mathcal{O}} L \leq 2 \chi \operatorname{diam}_{\mathcal{O}} K / \lambda^{n}$.

2.5. Sierpiński carpet Julia sets. Let us now assume additionally that the Julia set $\mathcal{J}_{R}$ of $R$ is a Sierpiński carpet. This means by definition that $\mathcal{J}_{R}$ is homeomorphic to the standard Sierpiński carpet. By Whyburn's characterization, a set $S \subset \widehat{\mathbb{C}}$ is a Sierpiński carpet if and only if it is compact, connected, locally connected, has no local cut-points, and has topological dimension 1 , see Why1]. This implies that each component of $\widehat{\mathbb{C}} \backslash S$ is a Jordan domain, distinct such complementary components have disjoint closures. Thus our Julia set $\mathcal{J}_{R}$ has these properties.

This means that each component of the Fatou set is a Jordan domain and that distinct components of the Fatou set have disjoint closures. Furthermore, the boundary of a component of the Fatou set cannot contain a critical value $v$, since any critical point $c$ with $R(c)=v$ would be a local cutpoint. The local connectedness of a continuum implies that it is an E-continuum (for any $\varepsilon>0$, only finitely many Fatou components have diameter larger than $\varepsilon$ ); see [Why2, Theorem VI.4.4]. We record this fact as a lemma for future reference.

Lemma 2.4. Let $\epsilon>0$ be arbitrary. Then there are only finitely many components $\Omega$ of $\mathcal{F}_{R}$ with $\operatorname{diam}(\Omega) \geq \epsilon$.

\section{REVIEW OF INVARIANT JORDAN CURVES}

Recall from the introduction that in $[\mathrm{BM}]$ the following result is given:

Theorem 3.1 ([BM, Theorem 14.1]). Let $f: S^{2} \rightarrow S^{2}$ be an expanding Thurston map. Then for each sufficiently large $n \in \mathbb{N}$ there is a Jordan curve $\mathcal{C} \subset S^{2}$ with $\operatorname{post}(f)$ that is invariant for the iterate $f^{n}$ of $f$.

Recall that this means that

$$
f^{n}(\mathcal{C}) \subset \mathcal{C}
$$

We will need a variant of this theorem. More precisely, we will need to adjust the proof in one specific place. Here an outline of the proof will be given, in the next section we will prove a slightly stronger version of this theorem.

A combinatorial condition that will allow us to construct the invariant curve is required.

Definition 3.2. Let $\operatorname{post}(f) \subset \mathcal{C} \subset S^{2}$ be a Jordan curve. A set $K \subset S^{2}$ joins opposite sides of $\mathcal{C}$ if

- $K$ intersects disjoint 0 -edges of $\mathcal{C}$ in the case when \#post $(f) \geq 4$;

- $K$ intersects all three 0 -edges of $\mathcal{C}$ in the case when \#post $(f)=3$. 
Recall that the 0 -edges of $\mathcal{C}$ are the closed arcs into which the postcritical points subdivide $\mathcal{C}$.

Definition 3.3. Let $f: S^{2} \rightarrow S^{2}$ be a Thurston map and $\mathcal{C} \subset S^{2}$ be an $f$-invariant Jordan curve with post $(f) \subset \mathcal{C}$. Then $f$ is combinatorially expanding for $\mathcal{C}$ if there is an $n \in \mathbb{N}$, such that no $n$-tile $X$ (defined in terms of $(f, \mathcal{C})$ ) joins opposite sides of $\mathcal{C}$.

If a Thurston map $f$ is expanding, the diameter of $n$-tiles goes to 0 as $n \rightarrow \infty$. Thus, an expanding Thurston map is always combinatorially expanding for any $f$-invariant Jordan curve $\mathcal{C}$.

The following theorem (which is [BM, Theorem 14.4 and Corollary 14.14]) is the main step in proving Theorem 3.1 .

Theorem 3.4. Let $f: S^{2} \rightarrow S^{2}$ be an expanding Thurston map. Assume there exist Jordan curves $\mathcal{C}, \mathcal{C}^{\prime} \subset S^{2}$ with $\operatorname{post}(f) \subset \mathcal{C}, \mathcal{C}^{\prime}$ and $\mathcal{C}^{\prime} \subset f^{-1}(\mathcal{C})$, and an isotopy $H: S^{2} \times[0,1] \rightarrow S^{2}$ rel. $\operatorname{post}(f)$ with $H_{0}=\mathrm{id}_{S^{2}}$ and $H_{1}(\mathcal{C})=\mathcal{C}^{\prime}$ such that the map

$$
\widehat{f}:=H_{1} \circ f \text { is combinatorially expanding for } \mathcal{C}^{\prime} .
$$

Then there exist an $f$-invariant Jordan curve $\widetilde{\mathcal{C}} \subset S^{2}$ with $\operatorname{post}(f) \subset \widetilde{\mathcal{C}}$.

Furthermore there is a homeomorphism $h: S^{2} \rightarrow S^{2}$ satisfying the following:

$$
\begin{aligned}
& h(\operatorname{post}(f))=\operatorname{post}(f), \\
& h(\widetilde{\mathcal{C}})=\mathcal{C}^{\prime}, \\
& h \text { maps the 1-tiles, 1-edges, and 1-vertices for }(f, \widetilde{\mathcal{C}}) \text { to the } \\
& \quad 1 \text {-tiles, } 1 \text {-edges, and 1-vertices for }(f, \mathcal{C}) .
\end{aligned}
$$

The second statement basically says that the 1-tiles for $(f, \mathcal{C})$ divide the 0 -tiles for $\left(f, \mathcal{C}^{\prime}\right)$ in the same combinatorial fashion as the 1-tiles for $(f, \widetilde{\mathcal{C}})$ subdivide the 0 -tiles for $(f, \widetilde{\mathcal{C}})$. Thus by choosing the Jordan curves $\mathcal{C}$ and $\mathcal{C}^{\prime}$ in a certain way, we may ensure that the invariant curve $\widetilde{\mathcal{C}}$ has certain desired properties. This will be the theme of the next section.

The following proposition and lemma finishes the proof of Theorem 3.1 in conjunction with Theorem 3.4. We will be using the following notation. Let $\mathcal{C}$ be an oriented Jordan curve and $p, q \in \mathcal{C}$. Then $\mathcal{C}(p, q)$ denotes the closed arc on $\mathcal{C}$ from $p$ to $q$.

Proposition 3.5 ([BM , Proposition 11.7 and proof of Lemma 11.17]). Suppose $\mathcal{C}$ is an oriented Jordan curve in $S^{2}$ and $P \subset \mathcal{C}$ a set consisting of $n \geq 3$ distinct points $p_{1}, \ldots, p_{n}, p_{n+1}=p_{1}$ in cyclic order on $\mathcal{C}$. Then there exists $\delta>0$ and $\epsilon_{0}>0$ satisfying the following.

(1) Let $\mathcal{C}^{\prime}$ be another Jordan curve in $S^{2}$ passing through the points of $P$ in the same cyclic order as $\mathcal{C}$, and let $\mathcal{C}^{\prime}\left(p_{i}, p_{i+1}\right)$ be the arc on $\mathcal{C}^{\prime}$ with endpoints $p_{i}$ and $p_{i+1}$. If

$$
\mathcal{C}^{\prime}\left(p_{i}, p_{i+1}\right) \subset \mathcal{N}^{\delta}\left(\mathcal{C}\left(p_{i}, p_{i+1}\right)\right)
$$

for all $i=1, \ldots, n$, then there exists an isotopy $H_{t}$ on $S^{2}$ rel. $P$ such that $H_{0}=\mathrm{id}_{S^{2}}$ and $H_{1}(\mathcal{C})=\mathcal{C}^{\prime}$.

(2) If $K \subset S^{2}$ is a set with $\operatorname{diam}(K)<\epsilon_{0}$ then $K$ does not join opposite sides of $\mathcal{C}^{\prime}$.

Theorem 3.6 ([BM, Lemma 11.17]). Let $f: S^{2} \rightarrow S^{2}$ be an expanding Thurston map. Let $\mathcal{C} \subset S^{2}$ be an arbitrary Jordan curve with post $(f) \subset \mathcal{C}$ and $\delta>0$ be arbitrary. Then for each sufficiently large $n \in \mathbb{N}$ we can find a Jordan curve $\mathcal{C}^{\prime} \subset f^{-n}(\mathcal{C})$ that traverses the 
the postcritical points of $f$ in the same cyclic order as $\mathcal{C}$ and for any two postcritical points $p_{i}, p_{i+1}$ that are consecutive on $\mathcal{C}$ we have

$$
\mathcal{C}^{\prime}\left(p_{i}, p_{i+}\right) \subset \mathcal{N}^{\delta}\left(\mathcal{C}\left(p_{i}, p_{i+1}\right)\right)
$$

\section{INVARIANT JoRDAN CURVES THAT SUBDIVIDE SECTORS}

As we explained in the introduction, we will construct a quotient map $f$ from $R$ which will be expanding. We wish to consider a forward invariant curve for (an iterate of) $f$ and construct a forward-invariant curve within its lift. In general, this construction will not result in a Jordan curve going through the postcritical set of $R$, cf. Lemma 6.6. We will be need a slight variant of Theorem 3.1, which will be proved in this section.

Let $f: S^{2} \rightarrow S^{2}$ be an expanding Thurston map. Assume that the Jordan curve $\mathcal{C} \subset S^{2}$ with $\operatorname{post}(f) \subset \mathcal{C}$ is $f$-invariant. Then the $n$-tiles subdivide the 0 -tiles. This means that each $n$-tile is contained in exactly one 0 -tile. Let us fix a postcritical point $p$. There are exactly two 0-edges, $E, E^{\prime}$ incident to $p$. Given an $n \in \mathbb{N}$, let $e_{1}, \ldots, e_{N}$ be the $n$-edges incident to $p$, ordered cyclically around $p$. Note that $N=2 \operatorname{deg}\left(f^{n}, p\right)$. Thus $N$, i.e., the number of such $n$-edges, goes to $\infty$ as $n \rightarrow \infty$ if and only if $p$ is of Fatou-type. Since $\mathcal{C}$ is $f$-invariant, there are exactly two of these $n$-edges, say $e_{i}$ and $e_{j}$, that are contained in the 0-edges $E$ and $E^{\prime}$ respectively. Relabeling if necessary, we can assume that $e_{i}=e_{1} \subset E$.

Now, it is entirely possible that $e_{j}=e_{2}$ or that $e_{j}=e_{N}$. In this case there are no $n$ edges $e_{k}$ incident to $p$ contained in the sector between $E$ and $E^{\prime}$, respectively in the sector between $E^{\prime}$ and $E$. We then say that the sectors between $E$ and $E^{\prime}$ are not subdivided by the $n$-edges, otherwise we say that the sectors between $E$ and $E^{\prime}$ are subdivided by the $n$-edges. It should be pointed out, that is possible that $p$ is of Fatou-type and the sectors between $E$ and $E^{\prime}$ are not subdivided by $n$-edges for any $n \in \mathbb{N}$.

The following theorem however means that we can avoid this phenomenon if desired.

Theorem 4.1. Let $f: S^{2} \rightarrow S^{2}$ be an expanding Thurston map. Then for each sufficiently high iterate $F=f^{n}$ there is an $F$-invariant Jordan curve $\mathcal{C} \subset S^{2}$ with $\operatorname{post}(f) \subset \mathcal{C}$, that has the additional property that for any Fatou-type postcritical point $p$ the sectors between the 0 -edges incident to $p$ are subdivided by the n-edges.

Here " $n$-edges" are $n$-edges of $(f, \mathcal{C})$, i.e., 1 -edges for $(F, \mathcal{C})$. To prove the theorem above, we need to find a Jordan curve $\mathcal{C}^{\prime} \subset f^{-n}(\mathcal{C})$ satisfying the conditions from Theorem 3.4 with the following additional property. At each Fatou-type postcritical point $p$, the sectors between the 0-edges $E, E^{\prime} \subset \mathcal{C}^{\prime}$ incident to $p$ are subdivided by the $n$-edges. Equivalently, the $n$-edges $e$ and $e^{\prime}$ in $\mathcal{C}^{\prime}$ that are incident to $p$ have the property that in each of the sectors between them, there is at least one $n$-edge incident to $p$.

Let $\mathcal{C} \subset S^{2}$ be a Jordan curve with post $(f) \subset \mathcal{C}$, which we orient arbitrarily. Let $\delta>0$ and $\epsilon_{0}$ be the constants from Proposition 3.5 for $P=\operatorname{post}(f)$. Let $n \in \mathbb{N}$ be sufficiently large such that the diameter of each $n$-tile $X$ is smaller than $\epsilon_{0}$ and $\delta$,

$$
\operatorname{diam}(X)<\min \left\{\epsilon_{0}, \delta\right\} .
$$

Furthermore, we assume that $n \in \mathbb{N}$ is sufficiently large, so that according to Theorem 3.6 there is a Jordan curve $\mathcal{C}^{\prime} \subset f^{-n}(\mathcal{C})$ that traverses the postcritical points in the same cyclic order as $\mathcal{C}$ and for any two postcritical points $p_{i}, p_{i+1}$ that are consecutive on $\mathcal{C}$ we have $\mathcal{C}^{\prime}\left(p_{i}, p_{i+1}\right) \subset \mathcal{N}^{\delta}\left(\mathcal{C}\left(p_{i}, p_{i+1}\right)\right)$.

From Theorem 3.4 it follows that there is an $f^{n}$-invariant Jordan curve $\widetilde{\mathcal{C}} \subset S^{2}$ with $\operatorname{post}(f) \subset \widetilde{\mathcal{C}}$. 


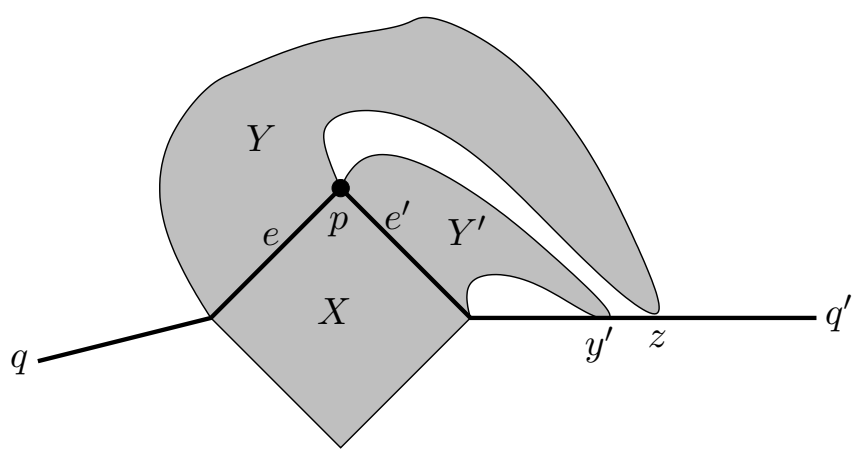

FIgURE 4.1. Constructing an $n$-edge path that subdivide sectors.

Now consider a Fatou-type postcritical point $p$. Clearly $\mathcal{C}^{\prime}$ contains two $n$-edges $e, e^{\prime}$ incident to $p$. In the case that there are other $n$-edges incident to $p$ in each of the two sectors between $e$ and $e^{\prime}$ it follows from the second statement in Theorem 3.4 that the $f^{n}$-invariant curve $\widetilde{\mathcal{C}}$ has the property that the sectors at $p$ are subdivided.

If the sectors between $e, e^{\prime}$ are not subdivided by $n$-edges we will adjust $\mathcal{C}^{\prime}$ in the following. We will ensure that we can still apply Proposition 3.5, and that the $f^{n}$-invariant curve resulting from Theorem 3.4 has the property that the $n$-edges subdivide the sectors at $p$.

Let us consider the union of all $n$-tiles containing $p$, i.e.,

$$
V^{n}(p):=\bigcup\left\{X \in \mathbf{X}^{n} \mid p \in X\right\} .
$$

Clearly, this is a closed set whose boundary is a union of $n$-edges. In the terminology of [BM], this is the closure of the $n$-flower of $p$.

Let $q$ and $q^{\prime}$ be the postcritical points that precede and succeed $p$ on $\mathcal{C}^{\prime}$. Let $a$ be the first point on $\mathcal{C}^{\prime}(q, p)$ that intersects $V^{n}(p)$, and $b$ be the last point on $\mathcal{C}^{\prime}\left(p, q^{\prime}\right)$ that intersects $V^{n}(p)$. Clearly $a$ and $b$ are $n$-vertices.

Lemma 4.2. In the setting as above assume that $\gamma(a, b)$ is an $n$-edge path contained in $V^{n}(p)$ with $p \in \gamma(a, b)$ that satisfies

$$
\gamma(a, b) \cap \mathcal{C}^{\prime}(q, a)=\{a\} \text { and } \gamma(a, b) \cap \mathcal{C}^{\prime}\left(b, q^{\prime}\right)=\{b\} .
$$

Then replacing $\mathcal{C}^{\prime}\left(q, q^{\prime}\right)$ by $\mathcal{C}^{\prime}(q, a) \cup \gamma(a, b) \cup \mathcal{C}^{\prime}\left(b, q^{\prime}\right)$ results in a Jordan curve $\mathcal{C}^{\prime \prime} \subset f^{-n}(\mathcal{C})$ that still satisfies the assumptions of Proposition 3.5 .

This means that $\mathcal{C}^{\prime \prime}$ traverses post $(f)$ in the same cyclical order as $\mathcal{C}$ and for postcritical points $p_{i}, p_{i+1}$ that are consecutive on $\mathcal{C}$ we have $\mathcal{C}^{\prime \prime}\left(p_{i}, p_{i+1}\right) \subset \mathcal{N}^{\delta}\left(\mathcal{C}\left(p_{i}, p_{i+1}\right)\right)$.

Proof. We first note that $\gamma(a, b)$ does not intersect any side $\mathcal{C}^{\prime}\left(p_{i}, p_{i+1}\right)$ that is distinct from $\mathcal{C}^{\prime}(q, p)$ and $\mathcal{C}^{\prime}\left(p, q^{\prime}\right)$. If this were the case, then any point in this intersection would be contained in an $n$-tile $X \subset V^{n}(p)$. Since $p \in X$, it follows that $X$ joins opposite sides of $\mathcal{C}^{\prime}$, which is a contradiction.

Since $\gamma(a, b)$ is in the $\delta$-neighborhood of $p \in \mathcal{C}$, it follows that $\mathcal{C}^{\prime \prime}(q, p) \subset \mathcal{N}^{\delta}(\mathcal{C}(q, p)$ and $\mathcal{C}^{\prime \prime}\left(p, q^{\prime}\right) \subset \mathcal{N}^{\delta}\left(\mathcal{C}\left(p, q^{\prime}\right)\right)$. This finishes the proof.

Lemma 4.3. Assume there are at least 4 -edges incident to $p$. Then there is an n-edge path $\gamma(a, b)$ that satisfies the assumptions of Lemma 4.2 and additionally that the two $n$ edges $e, e^{\prime} \subset \gamma(a, b)$ incident to $p$ have the property that in each of the two sectors between them there are other $n$-edges incident to $p$. 
Proof. The proof is illustrated in Figure 4.1. Let $e \subset \mathcal{C}^{\prime}$ and $e^{\prime} \subset \mathcal{C}^{\prime}$ be the $n$-edges in $\mathcal{C}^{\prime}$ that precede and succeed $p$ in $\mathcal{C}^{\prime}$. If each of the two sectors between $e$ and $e^{\prime}$ contains at least one $n$-edge incident to $p$, there is nothing to prove.

Assume now that one sector between $e$ and $e^{\prime}$ does not contain any $n$-edge incident to $p$. Then $e$ and $e^{\prime}$ are contained in a common $n$-tile $X$ incident to $p$, i.e., $X \subset V^{n}(p)$. Let $Y \subset V^{n}(p)$ be the $n$-tile distinct from $X$ that contains $e$, and $Y^{\prime} \subset V^{n}(p)$ be the $n$-tile distinct from $X$ that contains $e^{\prime}$. Since we assume that there are at least $4 n$-edges incident to $p$, it follows that $Y$ and $Y^{\prime}$ are distinct.

Let $y \in \partial Y$ be the first $n$-vertex in $\mathcal{C}^{\prime}(q, p)$ intersecting $Y$. Let $\gamma \subset \partial Y$ be the $n$-edge path in $Y$ between $y$ and $p$ that does not include $e$. Denote by $e_{Y}$ the last $n$-edge in $\gamma$, i.e., the $n$-edge in $\gamma$ incident to $p$.

Note that one sector between $e^{\prime}$ and $e_{Y}$ contains $e$, the other sector contains all other $n$-edges incident to $p$.

If $\gamma$ does not intersect $\mathcal{C}^{\prime}\left(p, q^{\prime}\right)$ we may replace $\mathcal{C}^{\prime}(y, p)$ by $\gamma$, finishing the desired construction.

Let us now assume that $\gamma$ does intersect $\mathcal{C}^{\prime}\left(p, q^{\prime}\right)$. The situation is illustrated in Figure 4.1. Let $z \in \partial Y$ be the first $n$-vertex on $\mathcal{C}^{\prime}\left(p, q^{\prime}\right)$ in this intersection. Then $\mathcal{C}^{\prime}(p, z)$ and the $n$-edge path on $\partial Y$ between $p$ and $z$ that does not contain $e$ form a Jordan curve that encloses $Y^{\prime}$. In particular $Y^{\prime}$ intersects $\mathcal{C}^{\prime}(q, p)$ only in $p$.

Now let $y^{\prime} \in \partial Y^{\prime}$ be the last $n$-vertex on $\mathcal{C}^{\prime}\left(p, q^{\prime}\right)$ intersecting $Y^{\prime}$. We now replace $\mathcal{C}^{\prime}\left(p, y^{\prime}\right)$ by the $n$-edge path $\gamma^{\prime}$ on $\partial Y^{\prime}$ between $p$ and $y^{\prime}$ that does not contain $e^{\prime}$. It follows that $\gamma^{\prime}$ intersects $\mathcal{C}^{\prime}(q, p)$ only in $p$. Let $e_{Y^{\prime}}$ be the $n$-edge in $\gamma^{\prime}$ incident to $p$. One sector between $e$ and $e_{Y^{\prime}}$ contains $e^{\prime}$. The other sector contains the other $n$-edges incident to $p$. This finishes the proof.

Proof of Theorem 4.1. Let $n \in \mathbb{N}$ be sufficiently large such that the assumptions stated in the beginning of this section are satisfied. Furthermore, we assume that any Fatou-type postcritical point is incident to at least $4 n$-edges.

Then from the Jordan curve $\mathcal{C}^{\prime}$ we can construct the Jordan curve $\mathcal{C}^{\prime \prime}$ by changing the curve in $V^{n}(p)$ for each Fatou-type postcritical point $p$ as in Lemma 4.3 . Theorem 3.4 finishes the proof.

Let $\mathcal{C} \subset S^{2}$ be an $f^{n}$-invariant Jordan curve with $\operatorname{post}(f) \subset \mathcal{C}$ such that the sectors between 0 -edges are subdivided by $n$-edges for some $n \in \mathbb{N}$ as given by Theorem 4.1. Note that for any iterate $f^{n k}$ of $f^{n}$ the Jordan curve $\mathcal{C}$ is again $f^{n k}$-invariant and the sectors at Fatou-type postcritical points are again subdivided by $n k$-edges.

\section{ExPANDING QUOTIEnTS}

In this and the following section we prove Theorem 1.2 . Let $R: \widehat{\mathbb{C}} \rightarrow \widehat{\mathbb{C}}$ be a postcritically finite rational map with Julia set homeomorphic to the Sierpiński carpet. Note that $R$ is not expanding in the sense of Definition 2.2. To be able to use Theorem 4.1, we take a quotient of $R$ that will turn out to be an expanding Thurston map.

Define an equivalence relation on $\widehat{\mathbb{C}}$ by $z \sim w$ if $z=w$ or if there is a Fatou component $\Omega$ such that $z, w \in \bar{\Omega}$. Since $\mathcal{J}_{R}$ is a Sierpiński carpet it follows that distinct Fatou components $\Omega$ and $\Omega^{\prime}$ have disjoint closures. So $\sim$ is indeed an equivalence relation. Let us denote by $\pi: \widehat{\mathbb{C}} \rightarrow \widehat{\mathbb{C}} / \sim$ the quotient map. Clearly $\pi$ is monotone, which means that for every point $x \in \widehat{\mathbb{C}} / \sim$ the set $\pi^{-1}(x) \subset \widehat{\mathbb{C}}$ is connected, since each equivalence class is connected. This implies that for any connected set $A \subset \widehat{\mathbb{C}} / \sim$ the set $\pi^{-1}(A) \subset \widehat{\mathbb{C}}$ is connected, see [Why2, VIII.2.2]. 
Theorem 5.1. In the setting as above we may identify $\widehat{\mathbb{C}} / \sim$ with $S^{2}$ so that the quotient map may be written as the continuous monotone map $\pi: \widehat{\mathbb{C}} \rightarrow S^{2}$. Furthermore there exists an expanding Thurston map $f: S^{2} \rightarrow S^{2}$ such that $f \circ \pi=\pi \circ R$.

We rely on the following notion and fact. An equivalence relation $\sim$ on the sphere $S^{2}$ is said to be of Moore-type, if

(1) $\sim$ is not trivial, meaning that there are at least two distinct equivalence classes;

$(2) \sim$ is closed (here we view the relation $\sim$ as a subset of $S^{2} \times S^{2}$ equipped with the product topology);

(3) each equivalence class is a compact connected set;

(4) no equivalence class separates $S^{2}$. This means that for any equivalence class $[x]$ of $\sim$ the set $S^{2} \backslash[x]$ is connected.

Moore's theorem is the fact that the quotient $\widetilde{S}^{2}:=S^{2} / \sim$ endowed with the quotient topology is homeomorphic to $S^{2}$ [Why2, Theorem IX.2.1'].

Now let $f: S^{2} \rightarrow S^{2}$ be a Thurston map. We call $\sim$ strongly $f$-invariant if the image of any equivalence class is an equivalence class, i.e.,

$$
f([x])=[f(x)],
$$

for any $x \in S^{2}$. The purpose of this condition is explained by the following, see [BM, Corollary 13.8] for a proof.

Lemma 5.2. Let $f: S^{2} \rightarrow S^{2}$ be a Thurston map and $\sim$ be a strongly $f$-invariant equivalence relation of Moore-type on $S^{2}$. Then there is a Thurston map $\widetilde{f}: \widetilde{S}^{2} \rightarrow \widetilde{S}^{2}$ such that

$$
\pi \circ f=\tilde{f} \circ \pi .
$$

The existence of $f$ follows essentially from the above in conjunction with Moore's theorem.

Lemma 5.3. There is a Thurston map $f: S^{2} \rightarrow S^{2}$ such that $f \circ \pi=\pi \circ R$.

Proof. According to Lemma 5.2 , we need to show that $\sim$ is of Moore-type and strongly $R$-invariant.

Clearly $\sim$ is not trivial. Furthermore each equivalence class is either a single point or a closed Jordan domain, thus compact, connected, and it does not separate $\widehat{\mathbb{C}}$.

To prove that $\sim$ is closed it suffices to show that given two convergent sequences $\left(x_{n}\right)_{n \in \mathbb{N}}$ and $\left(y_{n}\right)_{n \in \mathbb{N}}$ in $\widehat{\mathbb{C}}$ with $x_{n} \sim y_{n}$ for all $n \in \mathbb{N}$ it follows that $\lim x_{n} \sim \lim y_{n}$. This is clear in the case when for sufficiently large $n \in \mathbb{N}$ the points $x_{n}$ are contained in some fixed equivalence class, since each equivalence class is compact. Otherwise, we may assume that for distinct $n, m \in \mathbb{N}$ the points $x_{n}$ and $x_{m}$ are contained in distinct equivalence classes. In this case Lemma 2.4 shows that $\lim x_{n}=\lim y_{n}$. Thus $\sim$ is closed. We conclude that $\sim$ is of Moore-type. Thus $\widehat{\mathbb{C}} / \sim$ is homeomorphic to $S^{2}$, so we may identify the two spaces.

Note that if $x \in \widehat{\mathbb{C}}$ is not contained in the closure of a component of the Fatou set, the same it true for $R(x)$. Furthermore $R$ maps the closure of each component of the Fatou set to the closure of a component of the Fatou set. Thus $\sim$ is strongly $R$-invariant. Hence $f: S^{2} \rightarrow S^{2}$ is a Thurston map that satisfies $f \circ \pi=\pi \circ R$ by Lemma 5.2 .

So it remains to show that $f$ is expanding. We will need some preparation.

The set $F=\pi\left(\mathcal{F}_{R}\right)$ is countable. Let us say that an open set $U$ in $\widehat{\mathbb{C}}$ is clean if $\partial U \cap \bar{\Omega}=\emptyset$ for all Fatou components $\Omega$. If $U$ is a clean neighborhood it is saturated with respect to $\sim$ and the restriction $\left.\pi\right|_{\partial U}$ is a homeomorphisms onto its image. 
Lemma 5.4. Let $\xi$ be an equivalence class. For any open subset $U$ containing $\xi$, there exists a clean open set $V$ with boundary a simple closed curve such that $\xi \subset V \subset U$.

Proof. Identify $\pi(\widehat{\mathbb{C}})=S^{2}$ with the Euclidean sphere. Since $S_{r}=\left\{x \in S^{2}, d(\pi(\xi), x)=r\right\}$, $r>0$, is an uncountable family of pairwise disjoint sets and $F\left(=\pi\left(\mathcal{F}_{R}\right)\right)$ is countable, we may find $\left(r_{n}\right)$ tending to 0 such that $S_{r_{n}} \cap F=\emptyset$ The set $V_{n}=\pi^{-1}\left(\left\{x \in S^{2}, d(\pi(\xi), x)<\right.\right.$ $\left.\left.r_{n}\right\}\right)$ is open and clean by construction. Furthermore,

$$
\cap V_{n}=\pi^{-1}\left(\cap\left\{x \in S^{2}, d(\pi(\xi), x)<r_{n}\right\}\right)=\xi
$$

holds so that for $n$ large enough, one has $\xi \subset V_{n} \subset U$.

Lemma 5.5. For any $x \in S^{2}$ there is a neighborhood $V \subset S^{2}$ of $x$ such that the maximal diameter of any component of $f^{-n}(V)$ tends to 0 as $n \rightarrow \infty$.

Proof. By the shrinking lemma, one may find an open simply connected neighborhood $U_{z} \subset \widehat{\mathbb{C}}$ for each $z \in \mathcal{J}_{R}$ such that the components of $R^{-n}\left(U_{z}\right)$ are as small as wanted provided $n$ is large enough. Since $R$ is postcritically finite, either $z \notin \operatorname{post}(R)$ and so we may choose $U_{z}$ disjoint from $\operatorname{post}(R)$ or $z \in \operatorname{post}(R)$ and so we may assume $U_{z} \cap \operatorname{post}(R)=\{z\}$.

Let $x \in S^{2}$ be arbitrary and set $\xi=\pi^{-1}(\{x\})$ so that $\xi \subset \widehat{\mathbb{C}}$ is an equivalence class. Let $U_{\xi}^{\prime}$ be the union of $\xi$ and the union of all $U_{z}$ 's for $z \in \xi \cap \mathcal{J}_{R}$ (note that if $\xi=\{z\}$ is a singleton then $U_{\xi}^{\prime}=U_{z}$ ). According to Lemma 5.4, we may find a clean and simply connected open set $U_{\xi}$ such that $\xi \subset U_{\xi} \subset U_{\xi}^{\prime}$. Since $\mathcal{J}_{R}$ is a carpet, $U_{\xi}$ contains at most one point from $\operatorname{post}(R)$ which is contained in $\xi$, see Section 2.5 .

Endow $S^{2}$ with a metric compatible with its topology and let $\varepsilon>0$; the uniform continuity of $\pi$ implies the existence of $\delta>0$ such that sets of diameter less than $\delta$ are mapped under $\pi$ to sets of diameter at most $\varepsilon / 2$. By choosing $n$ large enough, we can ensure that each of the components $W$ of $R^{-n}\left(U_{\xi}\right)$ will be covered by the union of a single preimage $\zeta$ of $\xi$ and of components of $R^{-n}\left(U_{z}\right), z \in \xi$, each of which intersects $\zeta$ and has diameter at most $\delta$. Thus $\operatorname{diam} \pi(W) \leq \varepsilon$.

Now define $V:=\pi\left(U_{\xi}\right) \subset S^{2}$. Since $U_{\xi}$ is saturated and open, it follows that $V$ is an open neighborhood of $x \in S^{2}$. Let $V^{\prime} \subset S^{2}$ be a component of $f^{-n}(V)$. Since $\pi$ is monotone, it follows that $\pi^{-1}\left(V^{\prime}\right) \subset \widehat{\mathbb{C}}$ is connected. This implies in conjunction with the identity $f \circ \pi=\pi \circ R$ that $\pi^{-1}\left(V^{\prime}\right)$ is contained in a component of $R^{-n}\left(U_{\xi}\right)$. Thus $\operatorname{diam}\left(V^{\prime}\right) \leq \epsilon$ as desired.

Proof of Theorem 5.1. According to [BM, Proposition 6.3], the map $f$ is an expanding Thurston map if there is a finite covering $\mathcal{V}$ of $S^{2}$ by connected open sets such that $\left(\mathcal{V}_{n}\right)_{n}$ defines a basis for the topology of $S^{2}$ where $\mathcal{V}_{n}$ denotes the set of connected components of $f^{-n}(V)$ for $V \in \mathcal{V}$. Apply Lemma 5.5 and let $\mathcal{V}$ be a finite subcover. The theorem follows.

\section{LifTS OF JORDAN CURVES}

We are now ready to prove Theorem 1.2 . Let $R: \widehat{\mathbb{C}} \rightarrow \widehat{\mathbb{C}}$ be a postcritically finite rational map with Sierpiński carpet Julia set. From Theorem 5.1 we obtain the expanding Thurston map $f: S^{2} \rightarrow S^{2}$ and the monotone map $\pi: \widehat{\mathbb{C}} \rightarrow S^{2}$ such that $f \circ \pi=\pi \circ R$. As in the last section we let $F:=\pi\left(\mathcal{F}_{R}\right)$.

The idea to construct the $R^{n}$-invariant Jordan curve $\Gamma \subset \widehat{\mathbb{C}}$ is to start with an $f^{n}$ invariant Jordan curve $\mathcal{C} \subset S^{2}$ given by Theorem 4.1 and extract an $R^{n}$-invariant Jordan curve in $\pi^{-1}(\mathcal{C})$. By the remark at the end of Section 4, we may take further iterates 
$f^{n k}$ (and correspondingly $R^{n k}$ ) if convenient. To simplify the discussion, we assume in the following however that $n=k=1$.

Theorem 6.1. With the above notation, let $\mathcal{C} \subset S^{2}$ be an $f$-invariant Jordan with $\operatorname{post}(f) \subset$ $\mathcal{C}$, such that at each Fatou-type postcritical point the sectors between 0 -edges are subdivided by 1 -edges. Then there exists an $R$-invariant Jordan curve $\Gamma \subset \widehat{\mathbb{C}}$ satisfying $\operatorname{post}(R) \subset \Gamma$ as well as $\pi(\Gamma)=\mathcal{C}$.

Note that if $\mathcal{C} \subset S^{2}$ is any Jordan curve, there is in general no Jordan curve $\Gamma \subset \widehat{\mathbb{C}}$ with $\pi(\Gamma)=\mathcal{C}$.

Example 6.2. Consider the closed unit disk $\overline{\mathbb{D}} \subset \mathbb{C}$. Let $\gamma, \gamma^{\prime} \subset \mathbb{C} \backslash \overline{\mathbb{D}}$ be two disjoint arcs with the following properties. The arc $\gamma$ starts at the point $-2 \in \mathbb{R} \subset \mathbb{C}$ and winds infinitely often around $\overline{\mathbb{D}}$, so that $\gamma$ accumulates on $\partial \mathbb{D}$. Similarly $\gamma^{\prime}$ starts at $2 \in \mathbb{R} \subset \mathbb{C}$ and winds infinitely often around $\overline{\mathbb{D}}$ so that it accumulates on $\partial \mathbb{D}$.

Collapse the set $\overline{\mathbb{D}}$ to a point. More precisely, we consider the equivalence relation on $\mathbb{C}$ where $\overline{\mathbb{D}}$ is an equivalence class, all other equivalence classes are of the form $\{z\}$ where $z \in \mathbb{C} \backslash \overline{\mathbb{D}}$. Let $\pi: \mathbb{C} \rightarrow \mathbb{C} / \sim$ be the quotient map. It is easy to see that $\pi\left(\gamma \cup \overline{\mathbb{D}} \cup \gamma^{\prime}\right)$ is an arc, yet there is no Jordan arc contained in $\gamma \cup \overline{\mathbb{D}} \cup \gamma^{\prime}$ connecting -2 and 2 .

We start with several lemmas.

Lemma 6.3. Let $\mathcal{C} \subset S^{2}$ be a Jordan curve. Then $\widehat{\mathbb{C}} \backslash \pi^{-1}(\mathcal{C})$ has exactly two components.

Proof. Let $U_{1}$ and $U_{2}$ be the two components of $S^{2} \backslash \mathcal{C}$. Since $\pi$ is monotone, the sets $V_{1}:=\pi^{-1}\left(U_{1}\right) \subset \widehat{\mathbb{C}}$ and $V_{2}:=\pi^{-1}\left(U_{2}\right) \subset \widehat{\mathbb{C}}$ are connected. If $V_{1} \cup V_{2}$ would be connected, it would follow that $U_{1} \cup U_{2}=\pi\left(V_{1} \cup V_{2}\right)$ is connected, which is a contradiction.

To avoid problems as in Example 6.2, we will use that $\mathcal{C}$ is $f$-invariant and the shrinking lemma of the orbifold metric for $R$ in an essential way.

In the next lemma we consider a Jordan arc $c:[0,1] \rightarrow S^{2}$ starting at $x=c(0)$ that is invariant for $f$ in the following sense. We have $f(x)=x$ and there is an $s \in(0,1)$ such that $f(c([0, s]))=c([0,1])$.

Lemma 6.4. Let $c:[0,1] \rightarrow S^{2}$ be a Jordan arc that is invariant for $f$ as above and assume that $c(0,1] \cap \operatorname{post}(f)=\emptyset$. Then $\pi^{-1}(c(0,1])$ has a single accumulation point which is a fixed point for $R$.

Furthermore if $c^{\prime}:[0,1] \rightarrow S^{2}$ is a preimage of $c$ by some other iterate $f^{\ell}$, i.e., $c^{\prime}$ is another Jordan arc such that $f^{\ell}\left(c^{\prime}\right)=c$, then $\pi^{-1}\left(c^{\prime}(0,1]\right) \subset \widehat{\mathbb{C}}$ has a single accumulation point as well.

Proof. Using Lemma 5.5 and considering an iterate of $f$ if necessary, we may find a neighborhood $V \subset S^{2}$ with the properties that $x$ is the only possible postcritical point in $V$ and that $W=f^{-1}(V) \cap V$ is compactly contained in $V$. Pick $y=c(t) \in W \backslash F$ with $t>0$, such that $c[0, t] \subset W$ and denote by $c_{0}$ the closure of the connected component of $c \backslash\{y, f(y)\}$ bounded by $\{y, f(y)\}$. Define inductively $\left(c_{n}\right)_{n>0}$ so that $c_{n+1}$ is the lift of $c_{n}$ by $f$, where $c_{n} \cap c_{n+1}$ is the singleton $\left\{y_{n}\right\}$ such that $f^{n}\left(y_{n}\right)=y$. It follows that $\cup_{n \geq 1} c_{n}=c(0, t]$. Let $\kappa_{n}=\pi^{-1}\left(c_{n}\right)$, for each $n \geq 0$. Since $\pi$ is monotone, $\kappa_{n}$ is connected and $\kappa_{n+1} \cap \kappa_{n}$ is a singleton which is eventually mapped by $R^{n}$ to $\pi^{-1}(y)$. Note that $\cup_{n} \kappa_{n}=\pi^{-1}(c(0, t])$ is connected, again by the monotonicity of $\pi$.

It follows from [Dav, Lemma 17.3] that $\kappa_{0}$ is contractible, so that we may include it into a simply connected open set $D_{0} \subset W$ avoiding post $(R)$. For each $n \geq 1$, we may define an inverse branch $R_{-n}: D_{0} \rightarrow \mathcal{O}$ so that $R_{-n}\left(\kappa_{0}\right)=\kappa_{n}$. Since $D_{0}$ intersects $\mathcal{J}_{R}$, 
the sequence $\left\{R_{-n}\right\}_{n}$ is a normal family and any limit is constant. Therefore, we may find $n_{0}$ such that $\operatorname{diam}_{\mathcal{O}} \kappa_{n_{0}} \leq \delta$. By the shrinking lemma, we have $\operatorname{diam}_{\mathcal{O}} \kappa_{n} \lesssim \operatorname{diam}_{\mathcal{O}} \kappa_{0} / \lambda^{n}$ for all $n \geq 1$. This implies that $\operatorname{diam}_{\mathcal{O}} \cup_{n \geq k} \kappa_{n} \lesssim \lambda^{-k}$. Hence $\left(\kappa_{n}\right)_{n}$ converges to a single point $z \in \pi^{-1}(\{x\})$. Since $R\left(\kappa_{n}\right)=\kappa_{n-1}$ converges to $z$ as well, it follows that $z$ is a fixed point of $R$.

To see the second statement we note that the set $\pi^{-1}\left(c^{\prime}(0,1]\right) \subset \widehat{\mathbb{C}}$ is connected and its cluster set is connected as well as contained in $\pi^{-1}\left(c^{\prime}(0)\right)$. Since $R$ is open and

$$
R^{\ell}\left(\overline{\pi^{-1}\left(c^{\prime}(0,1]\right)}\right) \subset \overline{\pi^{-1}(c(0,1])},
$$

it follows that $\pi^{-1}\left(c^{\prime}(0,1]\right)$ has a single accumulation point.

Corollary 6.5. Let $\mathcal{C} \subset S^{2}$ be an $f$-invariant Jordan curve, $x \in F \cap \mathcal{C}$, and $c:[0,1] \rightarrow \mathcal{C}$ be a Jordan arc based at $x$, meaning that $c(0)=x$. Then $\pi^{-1}(c(0,1]) \subset \widehat{\mathbb{C}}$ accumulates at a single point.

Proof. Since the point $x$ is preperiodic, there are $n \geq 0$ and $k \geq 1$ such that the arc $c_{n}:=f^{n} \circ c$ is $f^{k}$-invariant. From Lemma 6.4 it follows that $\pi^{-1}\left(c_{n}((0,1])\right) \subset \widehat{\mathbb{C}}$ has a single accumulation point. From the second part in this lemma it follows that $\pi^{-1}(c((0,1])) \subset \widehat{\mathbb{C}}$ has a single accumulation point as desired.

Now consider the two $\operatorname{arcs}$ on $\mathcal{C}$ incident to some point $x \in F \cap \mathcal{C}$. By the above, their preimages by $\pi$ accumulate both at a single point in the boundary of $\pi^{-1}(x)$, which is the closure of a Fatou component. In order to construct the desired Jordan curve $\Gamma$, these two accumulation points need to be different. This is in fact the case when at each Fatou-type postcritical $p$ the sectors between the 0-edges incident to $p$ are subdivided by the 1-edges, see Section 4 for the terminology. This is the reason we introduced this notion and proved Theorem 4.1.

Lemma 6.6. Let $\mathcal{C} \subset S^{2}$ be an $f$-invariant Jordan curve with $\operatorname{post}(f) \subset \mathcal{C}$ such that the sectors between 0 -edges of every Fatou-type postcritical point is subdivided by 1-edges. Then for any $x \in F \cap \mathcal{C}$ the set $\pi^{-1}(\mathcal{C} \backslash\{x\})$ accumulates in $\pi^{-1}(x)$ in two distinct points.

Proof. Assume first that $x=p \in S^{2}$ is a periodic Fatou-type postcritical point of $f$. Let $\bar{\Omega}_{p}:=\pi^{-1}(p) \subset \widehat{\mathbb{C}}$. It is the closure of a Fatou component $\Omega_{p}$ of $R$ that contains a Fatou-type postcritical point of $R$.

Let $e, e^{\prime} \subset \mathcal{C}$ be the 1-edges incident to $p$. If $K:=\pi^{-1}(e \backslash\{p\})$ and $K^{\prime}:=\pi^{-1}\left(e^{\prime} \backslash\{p\}\right)$ accumulate at distinct points in $\partial \Omega_{p}$ we are done. So let us assume from now on that these sets both accumulate at the same point $z \in \partial \Omega_{p}$.

By assumption there is a 1-edge $e_{0}$ between $e$ and $e^{\prime}$ and a 1-edge $e_{1}$ between $e^{\prime}$ and $e$. Let $\widehat{e}_{0}$ and $\widehat{e}_{1}$ be the interiors of these 1-edges, i.e., we have removed the two endpoints from $e_{0}$ as well as $e_{1}$. Then $\widehat{e}_{0}$ and $\widehat{e}_{1}$ are disjoint. Let $z_{0}$ and $z_{1}$ be the accumulation points of $K_{0}:=\pi^{-1}\left(\widehat{e}_{0}\right)$ and $K_{1}:=\pi^{-1}\left(\widehat{e}_{1}\right)$ in $\partial \Omega_{p}$ respectively. Lemma 6.3 implies that $K_{0}$ and $K_{1}$ are in distinct components of $\pi^{-1}\left(S^{2} \backslash \mathcal{C}\right)$. Note that since $K$ and $K^{\prime}$ accumulate at the same point of $z \in \bar{\Omega}_{p}$, it follows that the closure of $\pi^{-1}(\mathcal{C} \backslash\{x\})$ separates $\Omega_{p}$ from $K_{0}$ or $K_{1}$. Without loss of generality, we may assume that it is $K_{0}$. This means that $z=z_{0}$.

Note that $f(e), f\left(e^{\prime}\right), f\left(e_{0}\right)$ are 0 -edges incident to $q=f(p)$. Since there are only two distinct such 0-edges, it follows that at least two of them have to coincide; let us assume $f(e)=f\left(e^{\prime}\right)$. Hence, $\pi^{-1}(f(e) \backslash\{q\})=\pi^{-1}\left(f\left(e^{\prime}\right) \backslash\{q\}\right)$ holds as well. But since $f \circ \pi=\pi \circ R$, it follows that $R(K)=R\left(K^{\prime}\right)$. But this contradicts the fact that $R$ is one-to-one in a neighborhood of $z$. This contradiction shows that $z \neq z^{\prime}$ as desired. 
Now let $x \in F$ be arbitrary. Then there is an $k \in \mathbb{N}$ such that $p:=f^{k}(x)$ is a periodic postcritical point. The two $(k+1)$-edges $e, e^{\prime} \subset \mathcal{C}$ incident to $x$ are mapped to (possibly the same) 1-edges in $\mathcal{C}$ incident to $p$. Thus, the $(k+1)$-edges incident to $x$ divide the sectors between $e$ and $e^{\prime}$. The argument now proceeds exactly as above.

We are now ready to prove Theorem 6.1

Proof of Theorem 6.1. Let $\mathcal{C} \subset S^{2}$ be an $f$-invariant Jordan curve with post $(f) \subset \mathcal{C}$, such that at each Fatou-type postcritical point the sectors between 0-edges are subdivided by the 1-edges as in Section 4 . Let $K:=\pi^{-1}(\mathcal{C}) \subset \widehat{\mathbb{C}}$ and $L \subset \widehat{\mathbb{C}}$ be the closure of $\pi^{-1}(\mathcal{C} \backslash F)$. Note that for any Fatou component $\Omega \subset K$, the set $L \cap \bar{\Omega}=L \cap \partial \Omega$ consists of exactly two distinct points by Lemma 6.6 .

We let $\Gamma \subset \widehat{\mathbb{C}}$ be the union of $L$ and the set of internal rays which join a point of $L \cap \partial \Omega$ to the center of $\Omega$ for each Fatou component $\Omega \subset K$. Since the Fatou set $\mathcal{F}_{R}$ is invariant for $R$, it follows that $F \cap \mathcal{C}$ is forward invariant by $f$. From $f \circ \pi=\pi \circ R$ it follows that $L$ is forward invariant for $R$. Hence any internal ray in $\Gamma$ is mapped to another internal ray in $\Gamma$. Thus $\Gamma$ is (forward) $R$-invariant, meaning that $R(\Gamma) \subset \Gamma$.

Note that $\pi(\operatorname{post}(R))=\operatorname{post}(f)$ (see [BM, Theorem 13.6]). Thus post $(R) \subset \pi^{-1}(\operatorname{post}(f))$. So any $q \in \operatorname{post}(R)$ is contained in the set $\pi^{-1}(p)$ for some $p \in \operatorname{post}(f)$. Note that $\pi^{-1}(p)$ is either $\bar{\Omega}_{q} \subset K$, the closure of the Fatou component of $R$ whose center is $q$, or $\pi^{-1}(p)=\{q\} \subset L$. In any case $q \in \Gamma$ by construction, meaning that $\operatorname{post}(R) \subset \Gamma$. Finally, the fact that $\pi(\Gamma)=\mathcal{C}$ is obvious from the construction as well.

Claim. $\Gamma$ is a Jordan curve.

First, we define a retraction map $\psi: K \rightarrow \Gamma$ as follows. If $z \in L$, then set $\psi(z)=z$. For any Fatou component $\Omega \subset K$ there is monotone retraction $\rho: \bar{\Omega} \rightarrow \bar{\Omega} \cap \Gamma$ mapping the closed Jordan domain to the crosscut defined by $\Gamma \cap \bar{\Omega}$; we let $\psi=\rho$ there.

We claim that $\psi$ is a retraction. The continuity follows from the fact that $\mathcal{J}_{R}$ is an $E$ continuum (cf. p. 5). Moreover, $\psi$ is a retraction since it is the case on the closure of each Fatou component contained in $K$ and is the identity elsewhere. Therefore, $\Gamma$ is continuum.

According to [Why2, Theorem III.7], it is sufficient to prove that any pair of distinct points of $\Gamma$ separates $\Gamma$. Let us consider two points $\{z, w\} \subset \Gamma$ and set $x=\pi(z)$ and $y=\pi(w)$.

If $x=y$, then $z, w$ are in the closure of the same Fatou component $\Omega \subset K$, so it follows easily that $\Gamma \backslash\{z, w\}$ is disconnected. Let us now assume that $x \neq y$. Since $\mathcal{C}$ is a Jordan curve, $\{x, y\}$ separates $\mathcal{C}$, hence, since $\pi$ is continuous, $\left\{\pi^{-1}(x), \pi^{-1}(y)\right\}$ separates $K$ and, since $\psi$ is monotone, $\left\{\psi\left(\pi^{-1}(x)\right), \psi\left(\pi^{-1}(y)\right)\right\}$ separates $\Gamma$. Now, $I_{x}=\psi\left(\pi^{-1}(x)\right)$ and $I_{y}=\psi\left(\pi^{-1}(y)\right)$ are intervals which contain $z$ and $w$ respectively. If these are non degenerate, we may define retractions $\rho_{z}: I_{x} \backslash\{z\} \rightarrow \partial I_{x} \backslash\{z\}$ and $\rho_{w}: I_{y} \backslash\{w\} \rightarrow \partial I_{y} \backslash\{w\}$, yielding a monotone retraction $\rho: \Gamma \backslash\{z, w\} \rightarrow \Gamma \backslash\left(I_{x} \cup I_{y}\right)$ by letting $\rho=$ id on the complement. This enables us to conclude that $\{z, w\}$ separates $\Gamma$. Thus $\Gamma$ is a Jordan curve.

We are ready to finish the proof of our main theorem.

Proof of Theorem 1.2. Let $R: \widehat{\mathbb{C}} \rightarrow \widehat{\mathbb{C}}$ be a postcritically finite rational map whose Julia set is homeomorphic to the Sierpiński carpet. Let $f: S^{2} \rightarrow S^{2}$ be the expanding Thurston map as given by Theorem 5.1. From Theorem 4.1 it follows that for each sufficiently 
high iterate $f^{n}$ there is an $f^{n}$-invariant Jordan curve $\mathcal{C} \subset S^{2}$ with post $(f) \subset \mathcal{C}$, that has the additional property that for any Fatou-type postcritical point $p$ the sectors between the 0-edges incident to $p$ are subdivided by the $n$-edges. Note that $\operatorname{post}\left(f^{n}\right)=\operatorname{post}(f)$ and $\operatorname{post}\left(R^{n}\right)=\operatorname{post}(R)$. Applying Theorem 6.1 to $f^{n}$ and to this curve $\mathcal{C}$ finishes the proof.

Remark 6.7. In the case when $\mathcal{C} \subset S^{2}$ with post $(f) \subset \mathcal{C}$ is $f$-invariant, but does not necessarily divide sectors at Fatou-type postcritical points, the construction given in the proof of Theorem 6.1 still yields an $R$-invariant set $\Gamma \subset \widehat{\mathbb{C}}$ with $\operatorname{post}(R) \subset \Gamma$. The set $\Gamma$ however will not be a Jordan curve in general, but a Jordan curve together with countably many internal rays connecting the center of Fatou components to this Jordan curve. In fact, we may remove all but finitely many of these internal rays and still have an $R$-invariant set $\Gamma \subset \widehat{\mathbb{C}}$ with $\operatorname{post}(R) \subset \Gamma$.

\section{ACKNowledgments}

The authors thank Tan Lei, who did initiate this collaboration. We are grateful to Mario Bonk, Mikhail Hlushchanka, and Kevin Pilgrim for their comments on a preliminary version. Y.G and JS.Z thank Professor Cui for helpful suggestions and discussions. P.H. is partially supported by the ANR projects "GDSous/GSG" no. 12-BS01-0003-01 and "Lambda" no. 13-BS01-0002. D.M. has been partially supported by the Academy of Finland via the Centre of Excellence in Analysis and Dynamics Research (project No. 271983), as well as by the Deutsche Forschungsgemeinschaft (DFG-ME 4188/1-1). P.H and D.M. thank IPAM for their hospitality where part of this work has been done during the program "Interaction between Analysis and Geometry".

\section{REFERENCES}

[Ber] Jérôme Bernard. Dynamique des perturbations d'un exemple de Lattès. Ph.D. thesis, Université de Paris-Sud, Orsay, 1994.

[BLM] Mario Bonk, Misha Lyubich and Sergei Merenkov. Quasisymmetries of Sierpiński carpet Julia sets. Preprint, 2013.

[BM] Mario Bonk and Daniel Meyer. Expanding Thurston maps. available at http:users.jyu.fi/ idanmeyer/files/draft.pdf

[CFKP] James W. Cannon, William J. Floyd, Richard Kenyon and Walter R. Parry. Constructing rational maps from subdivision rules. Conform. Geom. Dyn. 7 (2003), 76-102 (electronic).

[CFP] James W. Cannon, William J. Floyd and Walter R. Parry. Constructing subdivision rules from rational maps. Conform. Geom. Dyn. 11 (2007), 128-136 (electronic).

[CGNPP] Kristin Cordwell, Selina Gilbertson, Nicholas Nuechterlein, Kevin M. Pilgrim and Samantha Pinella. On the classification of critically fixed rational maps. Conform. Geom. Dyn. 19 (2015), 51-94 (electronic).

[Dav] Robert J. Daverman. Decompositions of manifolds Pure and Applied Mathematics, 124. Academic Press, Inc., Orlando, FL, 1986.

[Dev] Robert L. Devaney. Singular perturbations of complex polynomials. Bull. Amer. Math. Soc. (N.S.) 50 (2013), no. 3, 391-429.

[DH] Adrien Douady and John Hubbard. Etude Dynamique des Polynomes Complexes, I, II. Publications Mathématiques d'Orsay (1984/5).

[Eps] Adam Epstein. Bounded hyperbolic components of quadratic rational maps. Ergodic Theory Dynam. Systems 20 (2000), no. 3, 727-748.

[HP09] Peter Haïssinsky and Kevin M. Pilgrim. Coarse expanding conformal dynamics. Astérisque, 325, 2009.

[LMS1] Russell Lodge, Yauhen Mikulich and Dierk Schleicher. Combinatorial properties of Newton maps. Preprint arXiv:1510.02761, 2015.

[LMS2] Russell Lodge, Yauhen Mikulich and Dierk Schleicher. A classification of postcritically finite Newton maps. Preprint, arXiv arXiv:1510.02771, 2015. 
[McM1] Curtis T. McMullen. The classification of conformal dynamical systems. In Current developments in mathematics, 1995 (Cambridge, MA), pages 323-360. Internat. Press, Cambridge, MA, 1994.

[McM2] Curtis T. McMullen. Automorphisms of Rational Maps. In Holomorphic Functions and Moduli I, Vol. 10, Mathematical Sciences Research Institute Publications, 31-60. New York, Springer, 1988.

[Mil1] John Milnor. Geometry and dynamics of quadratic rational maps. Experiment. Math. 2 (1993), no. 1, 37-83. With an appendix by the author and Tan Lei.

[Mil2] John Milnor. Dynamics in one complex variable. Friedr. Vieweg \& Sohn, Braunschweig, 1999. Introductory lectures.

[Ree1] Mary Rees. Views of parameter space: Topographer and Resident. Astérisque (2003), vi+418.

[Ree2] Mary Rees. Persistent Markov partitions for rational maps. Preprint arXiv:1306.6166, 2013.

[Ros] Bastien Rosetti. Sur la détermination des fractions rationnelles postcritiquement finies par des graphes planaires finis. Ph.D. thesis, Université Paul Sabatier, 2015.

[Why1] Gordon Thomas Whyburn. Topological characterization of the Sierpiński curve. Fund. Math. 45 (1958), 320-324.

[Why2] Gordon Thomas Whyburn. Analytic topology. American Mathematical Society Colloquium Publications, Vol. XXVIII. American Mathematical Society, Providence, R.I., 1963.

[Wit] Ben Wittner. On the deformation loci of rational maps of degree two. Ph.D. thesis, Cornell University, 1988 .

Yan Gao, Mathemaitcal School of Sichuan University, 610065, P.R.China.

E-mail address: gyan@scu.edu.cn

Peter Haïssinsky, Université d'Aix-Marseille, Institut de Mathématiques de Marseille (I2M), 39, rue Frédéric Joliot Curie 13453 Marseille Cedex 13 France.

E-mail address: phaissin@math.univ-toulouse.fr

Daniel Meyer, Department of Mathematics and Statistics, P.O.Box 35, Fi-40014 UniverSITY OF JYVÄSKYlä, Finland.

E-mail address: dmeyermail@gmail.com

Jinsong Zeng, Academy of Mathematics and Systems Science, Chinese Academy of Sciences, Beijing 100190 P.R. China.

E-mail address: zeng.jinsong@amss.ac.cn 